\title{
Research on Power Grid Contact Aging Prediction Based on Variable Modified Grey Model
}

\author{
Yi Zhou, Peng Xiao, Shunhe Jia, Jianfei Chen and Sheng Zhang* \\ School of electronic and optical engineering, Nanjing University of Posts and Telecommunications, 210046 Nanjing, China \\ ${ }^{*}$ Corresponding author
}

\begin{abstract}
Research of grid contact is a hot topics of electrical power system. Serious aging of grid contacts will bring accidents or even fire. At present, the main research method of contact aging is grey model. It has the characteristics of convenient calculation and high prediction accuracy. However, the locality of factor variables is not considered in Traditional single variable grey model. For the multivariate gray model, it has high arithmetic and its prediction accuracy is too low. For this purpose, a Variable revision gray model is put forward in this paper. Firstly, Key factor variables is extracted for Modified variable. Secondly, Single behavior variable grey model is used to revise multivariate gray model. Finally, the proposed variable modified grey model is used in Prediction of contact aging. The results show that: In a complex environment, prediction accuracy of variable modified grey model much better than Traditional forecasting model. Up to $95.3 \%$. Furthermore, the model can also be used in the occasion that Variable effects have a significant difference in multivariate prediction.
\end{abstract}

Keywords—contact aging; grey model; variable correction

\section{INTRODUCTION}

Grid is not only a carrier of power market, but also an infrastructure that is related to the national economy and the people's livelihood and involves national security and social stability. In addition to being affected by the characteristics of the contact metal materials, the power grid contacts are subject to long-term effects of voltage, ambient temperature, light intensity and humidity, and will gradually undergo aging. Aging may lead to serious power accidents and even fire. Therefore, it is very necessary to predict the contact aging in time.

It have made a lot of work in the grid contact aging predictions at home and abroad, someone use of electroacoustic pulse method of pre-judgment [1]; Someone use the gray model to predict [2]; Someone predict with timevarying probability model [3]. Electroacoustic pulse method is the use of multiple acoustic interaction, the linear superposition principle is established to predict; Gray model is to organize the original data into regular data generated columns to predict; Time-varying Probability Method use graphs to represent the joint probability distribution of timevarying model variables and to generate probabilistic sequences to predict. Although our grid monitoring system can measure the contact temperature, leakage current, light intensity, humidity and other information. The loss of contact to the environment cannot be detected among the various aging prediction models above. The gray prediction model has the characteristic of establishing the prediction model through a small amount of incomplete information. More in line with the actual contact aging forecast [4].

The traditional univariate gray model GM $(1,1)$ has the inability to take the forecasting factors into account, longterm prediction accuracy of the limitations; But, the multivariate gray model GM $(1, N)$ is too much for consideration, the accuracy of short-term forecast has been reduced a lot. And its computational efficiency is low. To this end, we have improved the traditional gray prediction model, propose a gray model of variable revision. First of all, we make the main distinction between the various factors, choosing the factor variables that have the most influence on the behavioral variables to amend the univariate gray model. Next the univariate prediction is used to correct the overall multivariate gray model. It will Make the prediction accuracy has been greatly improved.

The aging of the grid contacts is mainly caused by external factors and internal factors. Internal factors are mainly due to long-term contact plus load current heat caused by the aging. The aging of this condition is mainly reflected by the change of the current inside the contacts and the temperature of the contacts. External factors that lead to the aging of the grid contacts are mainly the weather changes throughout the year. We must consider these factors comprehensively. In this paper, we first consider the various external influence factors that affect the aging of contacts. Then the gray model of variable correction is applied to the prediction of grid contact aging. It has achieved good predictive results.

\section{INTRODUCTION TO TRADITIONAL GRAY PREDICTION MODEL}

The traditional gray prediction model regards the random variation as the gray quantity which changes in a certain range .It commonly use generate cumulative, cumulative generation, the average generation, the ratio of generation and other methods will be disorganized raw data sorted into regular data generated strong column [4]. At present, the gray model mainly has two types of univariate and multivariate. Its mathematical principle is as follows: 


\section{A. Introduction of Univariate Gray Model}

The essence of the traditional GM $(1,1)$ model is to operate the original data to generate data with certain rules, Then through the establishment of differential equation model to predict. The first-order albino differential equation for establishing the GM $(1,1)$ model is:

$$
\frac{d y^{(1)}(t)}{d(t)}+a x^{(1)}(t)=u
$$

$y^{(1)}(t)$ is the cumulative sequence of $x^{(1)}(t)$.

$$
\psi(1)(\tau)=\sum_{i=1}^{k} x^{(0)}(i)
$$

According to the gray model basic formula, The gray model predictive formula of $y^{(1)}(t)$ is:

$$
\hat{y}^{(1)}(k+1)=\left(y^{(0)}(1)-\frac{u}{a}\right) e^{-a u}+\frac{u}{a}
$$

$\hat{y}^{(1)}(k+1)$ is the predicted value,Its model is:

$$
\hat{y}^{(0)}(k+1)=\left(1-e^{a}\right)\left(y^{(0)}(1)-\frac{u}{a}\right) e^{-a k}
$$

In order to visually show the prediction effect. We calculate the variance of $y^{(0)}-\hat{y}^{(0)}$

$$
\begin{gathered}
S_{1}^{2}=\frac{1}{n} \sum_{k=1}^{n}\left[y^{(n)}(k)-\frac{1}{n} \sum_{k=1}^{n} y^{(0)}(k)\right]^{2} \\
S_{2}^{2}=\frac{1}{n} \sum_{k=1}^{n}\left[y^{(0)}(k)-\hat{y}^{(0)}(k)-\frac{1}{n} \sum_{k=1}^{n}\left[x^{(0)}(k)-\hat{x}^{(0)}(k)\right]^{2}\right.
\end{gathered}
$$

\section{B. Multivariate Gray Model Introduction}

The traditional multivariate gray model adds a number of factor variables based on the univariate model.Recorded as: GM $(1, N), Z_{1}^{(1)}$ is the Immediate mean sequence of
$X_{1}^{(1)} \cdot X_{1}^{(0)}(k)+a z_{1}^{(0)}(k)=\sum_{i=2}^{3} b_{i} X_{i}^{(0)}(k)$ Is GM $(1, \mathrm{~N})$ gray differential equation. Among them $b_{i} X_{i}^{(0)}(k)$ is the driving item, $b_{i}$ is the starting coefficient

$$
z^{(1)}(k)=0.5 X^{(1)}(k)+0.5 X^{(1)}(k-1)
$$

$$
B=\left[\begin{array}{l}
-z_{1}^{(1)}(2), X_{2}^{(1)}(2), X_{3}^{(1)}(2) \\
-z_{1}^{(1)}(3), X_{2}^{(1)}(3), X_{3}^{(1)}(3) \\
\cdots \\
-z_{1}^{(1)}(n), X_{2}^{(1)}(n), X_{3}^{(1)}(n)
\end{array}\right]
$$

$$
Y=\left[\begin{array}{l}
X^{(0)}(2) \\
X^{(0)}(3) \\
\cdots \\
X^{(0)}(n)
\end{array}\right]
$$

$\phi=\left[a, b_{2}, b_{3}, \ldots b_{n}\right]^{T}$ the drive factor $b_{i}$ reflects the i-th related factors on the dependent variable size and trend. If $b_{i}$ is a plus number. This shows that the relevant factors and dependent variables are positively correlated. On the contrary is negative correlation; simultaneously. The greater the absolute of $b_{i}$ value indicates that the relevant factors on the dependent variable greater impact.

Other algorithms of GM $(1, \mathrm{~N})$ are similar to GM $(1,1)$. There are still some fatal flaws in GM $(1, N)$ prediction. Its effect is not very well, its short-term forecasting errors are often large. The main reason is that the multivariate gray model contains one behavior variable and more than one factor variable. One of these factors may have a direct impact on other factors. This can lead to significant changes to one of the other variables. Predict short-term accuracy has a great impact.

\section{VARIABLE REVISION GRAY MODEL}

In view of the disadvantages of the traditional gray model, we univariate the multivariate gray model and establish the model by determining the order of the variables according to the relationship between the variables. Firstly, we need to analyze the correlation between variables, extract the main factors as the behavioral variables, and then sort the remaining variables for the primary and secondary variables to extract the key factor variables that directly affect the values of the behavioral variables. Univariate gray prediction of behavioral variables was performed and modified with key factor variables. Finally, we make multivariable gray 
prediction on the other variables. Since the measured prediction values are most affected by the main behavioral variables, Therefore, the single-variable gray model is used to correct the multivariate gray forecasting value. The revised forecast not only considers the influence of multiple factors on the forecasting content. It also grasped the major contradictions and rationally revised the originally unsatisfactory predictions.

We first establish the GM $(1,1)$ model of behavioral variables, It is the essence of the original data is calculated to generate data has a certain law, Then through the establishment of differential equation model, the data is predicted.

Time series with behavioral variables

$$
y(0)=y^{0}(1), y^{0}(2), \ldots y^{0}(n)
$$

Do a cumulative sum of the generated sequence

$$
\begin{gathered}
y(1)=y^{(1)}(1), y^{(1)}(2), \ldots y^{(1)}(n) \\
y^{(1)}(k)=\sum_{i=1}^{k} x^{(0)}(i)
\end{gathered}
$$

In order to further improve the prediction accuracy, we use the gray cycle residual method to correct the original model [5], define the residual sequence. $\mathcal{E}^{(0)}(t)=X^{0}(t)-{ }^{\wedge}(0)(t)(t=0,1,2 \ldots, n) \quad$ Residual sequence plus predictive sequence can be a series of new sequence formula: $y^{(0)}(t)=\hat{T}^{(0)}(t)+\min \left\{\left|\varepsilon^{(0)}(t)\right|\right\}$ Establish the corresponding GM prediction model.

The above equation into the differential equation available

$$
\hat{y}^{(0)}(t+1)=\left(y^{0}(1)-\frac{u}{a}\right) e^{-a t}+\frac{u}{a}
$$

Adverse cumulative cumulative reduction can be obtained:

$$
\hat{y}^{(0)}(t+1)=\hat{y}^{(1)}(t+1)-\hat{y}^{(1)}(t)
$$

At this point, a set of improved behavioral variables gray combination prediction sequence has been drawn. In order to visualize the degree of data prediction. We need to calculate the variance of $y^{(0)}-\hat{y}^{(0)}$.Use the variables that have the most influence on behavioral variables to correct their variance.

Random of $X^{(0)}(k)$ is very big. Using a gray model of this variable to modify a multivariate model can affect the accuracy. The random sequence $\left\{\boldsymbol{X}^{0}(k)\right\}$ is corrected by a specific factor variable. A new sequence $\{r(t)\}$ is constructed.

$$
r(t)=u^{*} x(t)+(1-u) r(t-1) \quad 0<u<1
$$

$$
S_{1}^{2}=\frac{1}{n} \sum_{k=1}^{n}\left[y^{(n)}(k)-\frac{1}{n} \sum_{k=1}^{n} y^{(0)}(k)\right]^{2}
$$

$$
S_{2}^{2}=\frac{1}{n} \sum_{k=1}^{n}\left[y^{(0)}(k)-\hat{y}^{(0)}(k)-\frac{1}{n} \sum_{k=1}^{n}\left[\hat{x}^{(0)}(k)-r(t)\right]\right]^{2}
$$

$\mathrm{x}(\mathrm{c})$ is the factor variable. $\mathrm{u}$ is any constant between 0 and 1. Since this factor variable has the greatest impact on our predicted behavioral variables and is the same as the behavioral variable. Therefore, we use this factor variable to modify the gray model of behavioral variables. The selection of factor variables should be based on the specific application. However, this factor variable will not be considered in the prediction of multivariate gray model.

Calculate the Ratio of test difference: $c=S_{2} / S_{1}$ The smaller the better of $\mathrm{c}$. Finally, we are to determine the size of the data to predict the effect of the Ratio of test difference.

Next, we make multivariable predictions, taking into account the behavioral variables and the various factor variables. In order to facilitate writing, we unified with $\mathrm{X}$ for each data sample.

$X_{2}^{(1)}$ and $X_{3}^{(1)}$ are the sequence 1-AGO of factor variables, Taking into account the fact that some factor variables change very little. Available $\sum_{i=2}^{N} b_{i} X_{i}^{(1)}(k)$ is a gray constant. Then GM $(1,1)$ gray equation differential analog:

$$
\hat{X}_{1}^{(1)}+a z_{1}^{(1)}(k)=\sum_{i=2}^{n} b_{i} X_{i}^{(1)}(k)
$$

The corresponding approximate time obtained by solving the whitening equation of $\mathrm{GM}(1, \mathrm{~N})$ model is: 


$$
{\hat{X_{1}}}^{(1)}(k+1)=\left[X_{1}^{(0)}(1)-\frac{1}{a} \sum_{i=2}^{n} b_{1} X_{1}^{(1)}(k+1) e^{-a k}+\frac{1}{a} \sum_{i=2}^{n} b_{1} X_{1}^{(1)}(k+1)\right]
$$

Use GM modeling method to find the $\hat{X}^{(1)}$. And do a cumulative reduction of $\hat{X}^{(1)}$ into $\hat{X}^{(0)}$, The same calculation of the residual sequence, and calculate the relative error and Ratio of test difference. It is similar to the GM $(1,1)$ model.

Relative error $=$ (predicted value - measured value) / measured value. The remaining operations are similar to those of the traditional gray model. The measured results combine the ratio of the error of the single gray model with that of the multivariate gray model. The final result is:

$$
c=\frac{1}{2}\left(c_{1}+c_{2}\right)
$$

\section{Application of Gray Model With VARIABLE Correction in Aging Prediction of POWER NETWORK CONTACTS}

In the contact aging forecast, the contact temperature is the most important factor that affects the prediction of aging. We make the contact temperature as the behavior variable to predict the single variable gray model. Factors such as light intensity, leakage current and humidity are taken as factor variables to predict multivariable gray model; Since the ambient temperature directly affects the contact temperature, it is the variable that has the greatest influence on the contact temperature in the external disturbance and it is also the same as the temperature unit. Therefore, the univariate gray model of behavioral variables is modified by using the ambient temperature as the key factor variable. In this paper, the variable-corrected gray model is used to predict the annual average temperature of the grid contacts. The accuracy of the proposed method is compared with that of the traditional univariate gray model, the residual modified univariate gray model and the traditional multivariate gray model.

This paper divides the prediction error into four levels: One level relative error of less than 5\%.Ratio of test difference less than 0.6. Two level relative error of less than $10 \%$.Ratio of test difference less than 0.65 . Three level relative error of less than $20 \%$.Ratio of test difference less than 0.75 . Four level relative error of less than $30 \%$.Ratio of test difference less than 0.8 .

\section{A. Contact Annual Average Temperature Forecast}

The data in this paper come from the annual average contact temperature of the national grid [9], Contact leakage measured at a university science building in Nanjing, average annual intensity of light in Nanjing, average annual humidity and temperature in Nanjing. According to the annual average contact temperature in 2012-2014 to forecast the average annual contact temperature in 2015-2017. And compared with the measured contact temperature. The forecast results are shown in Table I.

TABLE I. VARIABLE FACTORS AND ANNUAL AVERAGE CONTACT TEMPERATURE PREDICTION RESULTS

\begin{tabular}{|c|c|c|c|c|c|c|c|c|}
\hline year & $\begin{array}{c}\text { Measure } \\
\text { contact } \\
\text { temperature }\end{array}$ & $\begin{array}{c}\text { Outside } \\
\text { temperatur } \\
\mathrm{e}\end{array}$ & $\begin{array}{c}\text { Leakage } \\
\text { current } \\
\mathrm{uA}\end{array}$ & $\begin{array}{c}\text { Light } \\
\text { intensity } \\
\mathrm{klx}\end{array}$ & $\begin{array}{c}\text { humidi } \\
\text { ty } \\
\%\end{array}$ & $\begin{array}{c}\text { Predict } \\
\text { contact } \\
\text { temperature }\end{array}$ & $\begin{array}{c}\text { Relative } \\
\text { error }\end{array}$ & $\begin{array}{c}\text { Ratio of } \\
\text { test } \\
\text { difference }\end{array}$ \\
\hline 12 & 24.6 & 16.7 & 1.79 & 39 & 86 & & & \\
\hline 13 & 24.2 & 16.2 & 1.82 & 40 & 68 & & & \\
\hline 14 & 23.5 & 15.6 & 1.90 & 45 & 72 & & & \\
\hline 15 & 26.8 & 17.2 & 2.00 & 35 & 76 & 26.3 & 1.9 & 0.58 \\
\hline 16 & 25.6 & 16.6 & 2.20 & 38 & 69 & 26.5 & 3.5 & 0.62 \\
\hline 17 & 27.6 & 18.5 & 2.60 & 42 & 78 & 28.9 & 4.7 & 0.64 \\
\hline
\end{tabular}

It can be seen from Table 1 that the relative error between the predicted value of this method and the actual measured value is less than 5\%. Ratio of test difference less than 0.6. Its prediction accuracy is between first and second level.

\section{B. Variables Correction Gray Model and Other Model Prediction Accuracy Comparisons}

In order to verify the validity of the gray model prediction for the correction of variables, based on the original data in Table 1, The annual average temperature of contact for 17 years was predicted by using four kinds of models: traditional univariate gray model, traditional multivariate gray model, residual uncorrected univariate gray model, and gray variable model modified by this paper. And analyzed their error size, the results shown in Table 2. It can be seen that the prediction accuracy of gray model with variable correction is the highest, which can reach $95.3 \%$. The residual gray model with unimodal correction of variables reaches $95.1 \%$, and its prediction accuracy is obviously higher than the prediction accuracy of the other two prediction methods. 
TABLE II. COMPARISON OF THE ACCURACY OF THE FOUR PREDICTION MODELS

\begin{tabular}{|c|c|c|c|}
\hline model & $\begin{array}{c}\text { Relative } \\
\text { error }\end{array}$ & $\begin{array}{c}\text { Ratio of } \\
\text { test } \\
\text { differenc } \\
\text { e }\end{array}$ & $\begin{array}{c}\text { Model } \\
\text { order H }\end{array}$ \\
\hline $\begin{array}{c}\text { univariate } \\
\text { gray } \\
\text { model }\end{array}$ & $93.6 \%$ & 0.72 & $\mathrm{H}=1$ \\
\hline $\begin{array}{c}\text { Residual } \\
\text { error } \\
\text { correction } \\
\text { univariate } \\
\text { gray } \\
\text { model }\end{array}$ & $95.1 \%$ & 0.67 & $\mathrm{H}=1$ \\
\hline $\begin{array}{c}\text { multivaria } \\
\text { te gray } \\
\text { model }\end{array}$ & $91.3 \%$ & 0.76 & $\mathrm{H}=5$ \\
\hline $\begin{array}{c}\text { Variable } \\
\text { revision } \\
\text { gray } \\
\text { model }\end{array}$ & $95.3 \%$ & 0.63 & $\mathrm{H}=3$ \\
\hline
\end{tabular}

In addition, since the univariate gray model only predicts the temperature based on the contact temperature, the aging of the grid contact is obviously not only reflected in the temperature, but also related to the current size, light intensity, and environmental humidity. At the same time, the multivariate gray model has lower prediction accuracy. Therefore, this paper combines the advantages of the two variables modified gray model in the actual contact aging prediction should have better practical value.

\section{CONCLUSION}

In this paper, aiming at the low prediction accuracy and large limitation of the traditional gray model, a gray model based on variable modification is proposed. This model firstly modifies the univariate gray model of the behavioral variables with the key factor variables, and then uses the univariate gray model The gray model which has filtered the factor variables is modified. Finally, the validity of the gray model is validated by the relative error and the test-error ratio.

In this paper, the modified gray model is applied to predict the aging of the contact of the power grid. The prediction accuracy reaches $95.3 \%$. The performance is better than the traditional gray model and has a good reference value in the actual aging prediction of the grid contact.

\section{Acknowledgement}

The authors thank the anonymous reviewer and editors for their helpful comments and suggestions. This work supported by the 2018 2nd International Conference on Electrical Engineering and Automation.

\section{REFERENCES}

[1] Zhou Yuanxiang, Dai Chao, Huang Meng.Effect of aging temperature on the charge characteristics of oil-paper insulation during thermal aging [J] High Voltage Technology,2016,42(3): 868-875.

[2] Xue Songtao, Qian Yuyin, Wang Yuangong.Application of gray system model in structural damage identification [J]. Mechanics Quarterly,2003,4:528-533.
[3] WEI Wei, WANG En Risk assessment of grid dispatching operation under time-varying probabilistic model [J] Grid technology, 2013, 37 (12) :3509-3514.

[4] Zhang Ning, Han Fuchun, Wu Tianwen.Power System Load Forecasting Based on Gray Model [J] Electric Power Journal,2006,21(1):26-30.

[5] Liu Xiaodong, Zhang Wenwen, Wang Guibao. An Adjustable Gray Model for Power System Load Forecasting and Its Application [J]. Journal of North China Electric Power University,1991.

[6] Zhang Hongmei, Li Youguang.Aging Prediction of Surge Protector Based on Gray Model [J] Porcelain arrester,2016,6(274):73-76.

[7] Mohammad M.Barzegari, Ebrahim Alizadeh, Amir H. Pahnabi Greybox modeling and model predictive control forcascade-type PEMFC [M]. Contents lists available at ScienceDirect, 2017, 127:611-622.

[8] Jing Yea,Yaoguo Dang a, Bingjun Lib.Grey-Markov prediction model based on background valueoptimization and central-point triangular whitenization weightfunction [J] Contents lists available at ScienceDirect, 2018, 320-330.

[9] Chen Chunlin, Tu Zhengwei, Guo Liang. Practice of Network and Information Security Situation Awareness of State Grid Corporation [J]. Power Information and Communication Technology,2017,15(6):3-8. 